\title{
COOPERATIVAS VECINALES. UNA APROXIMACIÓN A LA GESTIÓN COLABORATIVA EN REHABILITACIÓN Y CONSERVACIÓN DE BARRIADAS
}

\author{
Carlos J. Rosa Jiménez \\ María José Márquez Ballesteros \\ Alberto E. García Moreno \\ iHTT (UMA)- Proyecto I+D+i RecoBA \\ Manuel R. García López \\ Arquitecto proyecto RecoBA
}

Artículo Recibido: 03/10/2015

Artículo Aceptado: 01/06/2016

\begin{abstract}
Resumen
El modelo económico y administrativo de la cooperativa de viviendas, desarrollado para la promoción de viviendas con condiciones ventajosas para sus propietarios, ha constituido un modelo de éxito en numerosas experiencias, un sistema capaz de eludir algunos problemas típicos de la promoción convencional. Sin embargo, la mayor parte de las cooperativas de promoción se extinguen una vez conseguido el objetivo de la obtención de las viviendas, por lo que el potencial del modelo colaborativo no se prolonga más allá de este hito.
\end{abstract}

Frente a esto, los modelos residenciales basados en el llamado co-housing (covivienda) suponen un paso más en el concepto de cooperativa, extendiendo su actividad al funcionamiento de los colectivos que los habitan. Así, los habitantes de una comunidad co-housing podrán disponer de beneficios como la obtención de servicios con precios y condiciones ventajosas, o la disposición de espacios de esparcimiento o actividad con bajos costes de adquisición y mantenimiento. En otras palabras, el modelo de co-housing intenta trasladar los aspectos colaborativos del modelo cooperativo al entorno y a la actividad de una comunidad de vecinos, implantando un modo de vida capaz de aprovechar el potencial del grupo frente al individuo.

Existen multitud de referentes históricos de estas ideas, desde las propuestas del socialismo utópico (Fourier, Godin) a las conocidas Unités d'habitation de Le Corbusier. Lo novedoso de las iniciativas contemporáneas de co-housing es su carácter bottom-up: modelos en los que el punto de partida es la comunidad, y desde ella surge la necesidad y la idea de la vida colaborativa. Por tanto, uno de los factores determinantes para el éxito de un co-housing es una fuerte cohesión social de grupo y un sentimiento de pertenencia al colectivo. Esto hace pensar, a menudo, que el coWPS RI-SHUR, n-3, 2016, vol.1, ISSN: 2387-1768 


\title{
WPSReview International on Sustainable
}

\author{
Housing and Urban Renewal (RI-SHUR)
}

housing está vinculado a determinados sectores ideológicos; en realidad, se trata de un modelo que trasciende esos tópicos y se ha mostrado como una alternativa muy apropiada para determinados grupos sociales.

El proyecto $\mathrm{I}+\mathrm{D}+\mathrm{i}$ Cooperativas vecinales. Modelo de gestión colaborativa en rehabilitación y conservación de barriadas intenta, entre otros objetivos, indagar en las posibilidades del cooperativismo y el co-housing como medio para la recuperación, rehabilitación y conservación de edificios y entornos urbanos.

\begin{abstract}
The economic and administrative model of cooperative housing -established for the creation of housing developments with advantageous conditions for their future owners- has been adopted as a successful model in numerous countries. However, most of cooperative promotions are extinguished once the goal of obtaining housing has been achieved, so that the potential of the collaborative model is not extended in time beyond this milestone.
\end{abstract}

The cohousing experiences mean an important step in the concept of cooperative activity, extending it to the functioning of the groups that inhabit them. Thus, the inhabitants of a cohousing community may provide benefits such as obtaining services with advantageous prices and conditions, and the provision of recreational areas with low acquisition and maintenance costs. In other words, the cohousing model attempts to transfer the collaborative aspects of the cooperative model onto the neighbors' activity, introducing a lifestyle able to exploit the potential of group versus individual.

There are many historical references of these ideas, from the proposals of utopian socialism (Fourier, Godin) to well-known Le Corbusier's Unités d'Habitation, but perhaps the novelty of contemporary experiences of cohousing is the fact that these are initiatives bottom-up, where the starting point is the community. Therefore, one of the determining factors for the success of cohousing is the existence of strong social cohesion of the collective and a sense of belonging to the group. This causes often tend to think that cohousing is linked to certain ideological sectors, but the reality is that this is a model that transcends these topics and has proven to be a very suitable alternative for certain social groups..

Palabras clave: rehabilitación; co-housing; cooperativas; economía social; resiliencia urbana; regeneración de barrios

Área temática: Arquitectura y urbanismo sostenibles (regeneración urbana y revitalización de barrios)

\section{Introducción}

Este artículo aborda la tarea de la regeneración urbana desde una perspectiva dirigida a la humanización de nuestro hábitat, priorizando las relaciones participativas de los ciudadanos en la solución de los problemas. Las acciones cooperativas y colaborativas son una tendencia indiscutiblemente emergente en todos los sectores laborales y sociales.

WPS RI-SHUR, n3, 2016, vol.1, ISSN: 2387-1768 
WPSReview International on Sustainable

Housing and Urban Renewal (RI-SHUR)

\begin{tabular}{|c|c|c|c|c|}
\hline Estado edificio & $<1951$ & 1951-1980 & $1981-2001$ & TOTAL \\
\hline Ruinoso & 11.124 & 5.034 & 1.154 & 17.312 \\
\hline Malo & 19.125 & 9.280 & 2.104 & 30.509 \\
\hline Deficiente & 61.005 & 53.446 & 12.804 & 127.255 \\
\hline Bueno & 254.189 & 664.731 & 668.322 & 1.587 .242 \\
\hline Total & 345.443 & 732.491 & 684.384 & 1.762 .318 \\
\hline $\begin{array}{l}\text { Porcentaje respecto } \\
\text { al total }\end{array}$ & $20 \%$ & $42 \%$ & $39 \%$ & $100 \%$ \\
\hline
\end{tabular}

Tabla 1. Estado de los edificios destinados principalmente a vivienda en Andalucía. Fuente Censo 2001, INE.

Nos encontramos ante el gran reto de gestionar la regeneración urbana integral en las barriadas construidas ente la décadas de 1950 y 1980, ya que suponen el $40 \%$ del parque residencial en España, y presentan un gran debate sobre su futuro. Dentro de la apuesta por modernización y cualificación de las edificaciones, acercándola a las necesidades y demandas técnicas de la actualidad, la accesibilidad se plantea como un reto fundamental, ya que es una de las grandes carencias de estas edificaciones, sobre todo la de las barriadas construidas en los cincuenta y primera mitad de los sesenta.

En Andalucía más de setecientos mil edificios se construyeron en estas décadas, atendiendo que en España un 68\% del parque residencia se corresponde con vivienda colectiva, existen en Andalucía casi medio millón de edificios en los que es necesario establecer unas pautas de actuación y gestión para propiciar su regeneración (VVAA, 2015a).

En la actualidad estamos inmersos en un proceso de fomento de las políticas de vivienda que hacen especial hincapié en la mejora de la ciudad existente, y la rehabilitación del parque residencial actual. Para ello es necesario hablar de reciclaje del edificio, aunque es evidente que nos encontramos con grandes barreras como la división horizontal, la necesidad del consenso en los vecinos y la financiación.

Por lo tanto este proyecto de investigación parte de los resultados y diagnósticos del proyecto anterior y recoge algunos de los objetivos que actualmente plantea el Plan de Vivienda del Plan de Infraestructuras, Transporte y Vivienda PITVI (2012-24).

El Ministerio de Fomento, además de establecer medidas de apoyo a la rehabilitación y el alquiler, profundiza en la investigación de otros instrumentos que ayuden a la reconversión y reactivación del sector, promoviendo una estrategia común sobre suelo y vivienda que tenga especialmente en cuenta el desarrollo de la actividad económica

WPS RI-SHUR, nำ, 2016, vol.1, ISSN: 2387-1768 


\title{
WPSReview International on Sustainable
}

\author{
Housing and Urban Renewal (RI-SHUR)
}

y el empleo sobre el suelo productivo. Especial interés reviste el Plan Estratégico para la Rehabilitación y la Eficiencia Energética de la Edificación, que cumplirá el mandato del artículo 4 de la Directiva 2012/27/UE, de acuerdo con el cual, España redacta en junio de 2014 la estrategia a largo plazo para la rehabilitación energética en el sector de la edificación, y así intentar movilizar inversiones en la renovación del parque nacional de edificios residenciales y comerciales, tanto público como privado, activando el sector de la construcción y favoreciendo su reconversión hacia fórmulas más sostenibles y duraderas en el tiempo.

Por esta situación normativa especial, que pretende impulsar la regeneración del tejido urbano y residencial y el estímulo de otras alternativas para favorecer el acceso a la vivienda, se hace necesaria una investigación con aplicación práctica sobre nuevos métodos de gestión de las barriadas y comunidades, capaces de proponer soluciones equitativas al problema residencial y favorecer situaciones proclives a la creación de un empleo sostenible y de calidad. Se trata de nuevas fórmulas de gestión participativas que sean capaces de generar oportunidades y ventajas superiores a las que se obtienen desde las comunidades de vecinos. Es decir, promover la rehabilitación integral desde los propios miembros de la comunidad que, frente a las posibilidades de gestoras privadas, se organizan para gestionar mejor su entorno. Pero, para ello, es preciso que el marco normativo favorezca esta situación, de ahí que sea primordial que en los planes de vivienda se contemple esta nueva posibilidad.

El modelo cooperativo, basado en una estructura empresarial ligada a una serie de valores cuya traslación al ámbito de la convivencia vecinal puede producir resultados muy interesantes: la igualdad, la democracia en la toma de decisiones y la horizontalidad en los procesos son principios que deben imperar en las comunidades habitacionales (VVAA, 2015b), y así se establece en la Ley de Propiedad Horizontal que prevé el mecanismo de las comunidades de vecinos como principal órgano de gestión que regula la convivencia vecinal.

En ese sentido, la comunidad de propietarios con frecuencia tiende a ser percibido como un instrumento de mínimos que intenta conciliar los intereses de los diferentes propietarios, por lo que en numerosas ocasiones su mayor función consiste en minimizar la fricción entre copropietarios de unas zonas comunes e instalaciones que intentan reducirse a niveles mínimos. De modo que la comunidad de propietarios garantiza un umbral mínimo de convivencia. Pero, ¿qué ocurre cuándo una comunidad desea sobrepasar ese mínimo y constituir un colectivo que apueste por la vida en común y la actividad conjunta para obtener un bien comunitario?

La co-vivienda o co-housing es heredera del modelo Andel de cooperativas de viviendas de amplio desarrollo en los países escandinavos desde la década de 1960 (Suschek-Berger, 2010). Consiste en la formación de una comunidad autogestionada que entiende el hecho de habitar como una actividad colectiva y cooperativa. En consecuencia, los espacios habitados por una co-vivienda, cuenta con una relación entre espacios comunitarios y espacios privados superior a la habitual. Además, se trata de un modelo que favorece la centralización de servicios y la implantación de 


\title{
WPSReview International on Sustainable
}

\author{
Housing and Urban Renewal (RI-SHUR)
}

equipamientos comunitarios, lo que aporta beneficios sociales y económicos a los cooperativistas y repercute en una mejora de su calidad de vida (Cameron, 2012).

La relación entre los diferentes habitantes de la co-vivienda puede ser diferente en función del grado de implicación de cada uno en la actividad comunal, pero es evidente que los vecinos de estas comunidades son individuos con un importante sentimiento de pertenencia al colectivo (Scotthanson, 2005).

La forma de gestión más frecuente en los casos de vivienda colaborativa es la de la cesión de uso, es decir, la vivienda pertenece a la cooperativa y cada habitante o familia adquiere el derecho de uso de una vivienda a cambio de una aportación económica recuperable.

El modelo de co-vivienda o co-housing constituye la traslación de algunos de los principales aspectos colaborativos del sistema cooperativo al ámbito vecinal, superando el esquema funcional y de gestión impuesto por la comunidad de vecinos. Introduce valores de la vida cooperativa aprovechando el potencial del grupo frente al individuo.

Existen multitud de referentes históricos sobradamente conocidos que se basan en estas idea de la vida comunitaria, desde las propuestas del socialismo utópico materializadas en los falansterios de Charles Fourier, o los familisterios de JeanBaptiste André Godin, a las unités d'habitation de Le Corbusier, pero quizá lo novedoso de las experiencias contemporáneas de co-vivienda sea el hecho de que se trata de iniciativas de abajo arriba (bottom-up), es decir, el punto de partida es la comunidad, y es de ésta de donde surge la necesidad y la idea de enfrentarse al habitar de una manera colaborativa. Los modelos utópicos de Fourier y de Godin siempre encontraron los mismos problemas: la casi imposibilidad de encontrar personas suficientemente comprometidas e interesadas en formar parte de ellos. Se trataba de modelos teóricos muy difícilmente trasladables a la realidad, y que en las contadas ocasiones en que dieron lugar a experiencias pioneras se tradujeron en estrepitosos fracasos. Por otra parte, el modelo de unidad habitacional de Le Corbusier, basado en el racionalismo que caracteriza la obra del arquitecto suizo (la máquina de habitar), también trata de imponer un modelo top-down, es decir, crear de cero una sociedad, una comunidad autosuficiente que cubra todas sus necesidades básicas -también "diseñadas" o planificadas por el arquitecto- en el propio edificio. El funcionamiento de las unités nunca llegó a ser el que se esperaba, y se convirtieron en poco tiempo en edificios de viviendas con diversos locales cerrados y sin uso, probablemente porque su implantación no respondía a las necesidades reales de la comunidad que los habitaba, o dicho en otras palabras, el programa de necesidades social había sido desarrollado al margen de sus futuros habitantes.

Estos ejemplos indican que en este tipo de experiencias es imprescindible la existencia de una fuerte cohesión social de grupo, y de un cierto sentimiento de pertenencia al colectivo. Esto hace que, a menudo, se tienda a pensar que el movimiento co-housing está vinculado a determinados sectores ideológicos o clichés de los llamados movimientos antisistema, tales como el de la comuna hippie o la casa okupa. Pero la realidad es que se trata de un modelo que trasciende esos tópicos y se WPS RI-SHUR, nํ, 2016, vol.1, ISSN: 2387-1768 


\title{
WPSReview International on Sustainable
}

\author{
Housing and Urban Renewal (RI-SHUR)
}

ha mostrado como una alternativa muy apropiada para determinados colectivos sociales. De hecho, el modelo co-housing se basa en uno de los principios fundamentales del capitalismo: el de la competitividad, ya que intentar sumar fuerzas para que una comunidad consiga mejores servicios a mejor precio, o utilizar la energía de su propio capital humano en su propio beneficio equivale a intentar crear una comunidad más competitiva (VVAA, 2008; Vallés, 2011).

En este sentido, la co-vivienda se basa en un modelo cooperativo perfectamente legislado y tipificado en el marco legal estatal y andaluz: la cooperativa de consumidores y usuarios. La comunidad se establece como un conjunto de consumidores que se asocian por sus intereses comunes -ya sean éstos la necesidad de espacios de trabajo u ocio, la prestación de servicios o la compra de bienes o productos - de modo que son capaces de alcanzarlos con un precio o unas condiciones mejores que las que cada cooperativista alcanzaría por separado. Este fundamento se ejemplifica perfectamente en los casos de cooperativas de personas mayores, en los un grupo de personas con intereses y necesidades análogos se establece como cooperativa, pudiendo disfrutar de servicios "a medida" -tales como transporte, servicios médicos, fisioterapia, catering o terapia ocupacional- que difícilmente la economía de un pensionista medio podría costear en su totalidad.

Con frecuencia las experiencias de co-vivienda -al menos en nuestro país- establecen un sistema en que la propiedad del conjunto de viviendas pertenece a la cooperativa, y los socios compran mediante una cuota el derecho al uso, que además es transmisible mediante herencia o venta. También tienen la opción de abonar mediante cuotas el uso de los espacios y servicios comunes, que dependerá del grado de utilización de los mismos.

\section{Objetivos}

El objetivo general es la búsqueda y análisis de experiencias relacionadas con la rehabilitación y basadas en el modelo cooperativo. A su vez también se incorporan y analizan experiencias de co-vivienda o co-housing, todo ello para elaborar una serie de conclusiones que se traslade a la creación de un modelo teórico (Moreno, 2015).

- Mejorar las condiciones de los tejidos urbanos ya existentes y la seguridad, habitabilidad y sostenibilidad de las viviendas y edificaciones de una manera práctica buscando nuevas formas de gestión económica y social.

- Promover la regeneración urbana integrada de barrios completos, partiendo de la experiencia del proyecto anterior e transformándolo en una nueva forma de gestión colaborativa integral.

- Atender las nuevas necesidades residenciales surgidas como consecuencia de los cambios económicos, sociales y demográficos ya sean de largo alcance, u otros más coyunturales, como pueda ser las necesidades derivadas por el rápido crecimiento del volumen de desahucios y el problema de las viviendas vacías. 


\section{WPSReview International on Sustainable \\ Housing and Urban Renewal (RI-SHUR)}

\section{Metodología. Búsqueda de modelos cooperativos.}

Se trata de investigar la definición del concepto y las modalidades alternativas de gestión cooperativa, a través de una revisión de las experiencias nacionales e internacionales y análisis de sus ventajas.

En una primera fase se ha llevado a cabo una revisión documental y bibliográfica de experiencias internacionales de cooperativas de rehabilitación y de experiencias de cohousing o co-vivienda. En primer lugar, es necesario sentar las bases de la sistematización de la información recopilada, de manera que constituya una información estructurada y fácilmente actualizable por los distintos investigadores, además de incorporar las valoraciones objetivas de cada uno de los casos.

\section{Registro de la documentación y bibliografía consultada}

\section{Sistematización de la información y categorización de los datos}
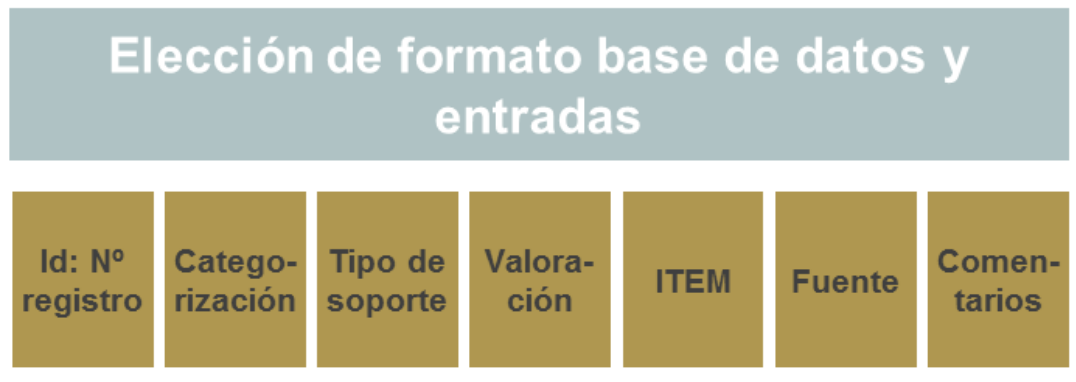

\section{- Consulta \\ abierta \\ - Fácilmente identificable \\ -Actualizable \\ - Información \\ valorada}

Figura 1. Proceso de sistematización de la información para la creación de la base de datos propia.

Para ello se creó una base de datos (Figura 1) organizada en 7 variables. El número de registro se corresponde con la identificación de referencia del dato. El tipo de soporte, ítem o fuente permite localizar la información. Los tres bloques elegidos para clasificar la información permiten categorizar las distintas entradas. Y por último, de cara a obtener resultados en esta fase documental, los indicadores que mejor contribuyen a la elaboración de los modelos de éxito son el de valoración y comentarios que se introduce en cada caso seleccionado, ya que conforme se actualiza la base documental también se iba estableciendo la utilidad relativa de cada uno de ellos.

En una segunda fase, se ha seleccionado aquellos casos que se pueden trasladar al modelo de gestión dirigido a la rehabilitación de barriadas. Si bien, el modelo WPS RI-SHUR, nํ, 2016, vol.1, ISSN: 2387-1768 


\section{WPSReview International on Sustainable \\ Housing and Urban Renewal (RI-SHUR)}

cooperativo de rehabilitación no debe basar su actividad exclusivamente en la fase de rehabilitación del proyecto, sino que debe de fomentar el desarrollo económico y social de la barriada. Por ello se incluyen ejemplos de experiencias de éxito algunos casos de cooperativas no vinculadas directamente a la vivienda, así como proyectos colaborativos y basados en la economía social de mayor escala que no responden exactamente a la estructura de la cooperativa. Se trata, por encima de todo, de buscar fuentes de ideas que puedan inspirar el catálogo de acciones que el nuevo modelo de cooperativa vecinal pueda emprender en su objetivo de regeneración a escala urbana y de la edificación.

Después del estudio de diversos modelos, se ha efectuado una selección de siete casos de estudio que contienen aspectos que se han considerado relevantes:

1. La vivienda colaborativa: Spreefeld, Berlín (Alemania)

2. La vivienda productiva: 60 Richmond East, Toronto (Cánada)

3. Cooperativa, rehabilitación y ayuda mutua: Covicivi, Montevideo (Uruguay)

4. La vivienda especializada: cooperativa Los Milagros, Málaga (España)

5. La regeneración cooperativa a escala urbana: Coin St., Londres (Reino Unido)

6. La resiliencia urbana: R-Urban, Colombes (Francia)

7. Cooperativa y energía: Zencer, Fuengirola (España)

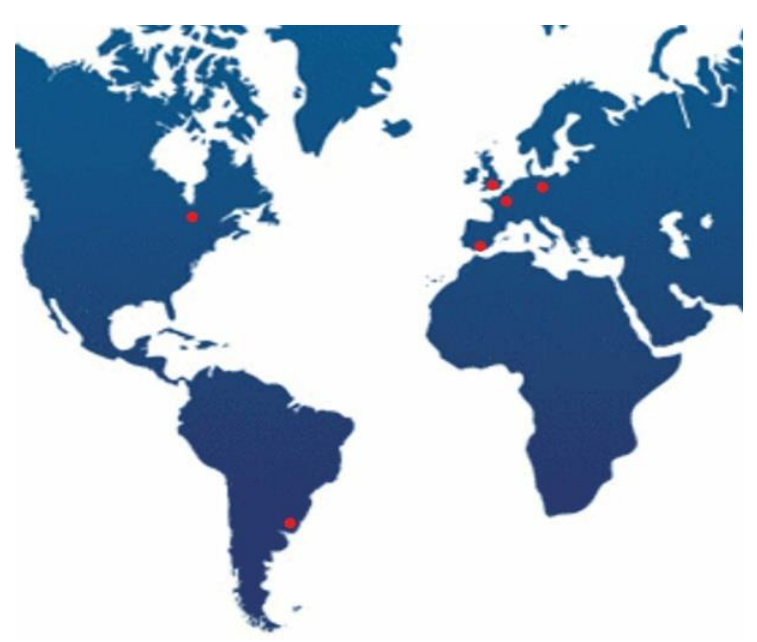

Figura 3. Localización de los casos de estudio relevantes seleccionados

Todos los casos de estudio tienen una característica común: se trata de procesos colaborativos que se basan en el modelo cooperativo con el fin de desarrollar proyectos o experiencias de manera democrática, horizontal y participativa. 


\section{WPSReview International on Sustainable \\ Housing and Urban Renewal (RI-SHUR)}

Como se puede observar en la figura 4, las principales aportaciones de los casos de estudio se han organizado en seis campos de intervención: espacios colectivos, vivienda, construcción/mantenimiento, energía, movilidad e impacto socio-económico.

\section{Resultados. Modelos internacionales de cooperación con relevancia para la rehabilitación de barriadas}

En lo que se refiera a los espacios colectivos se caracterizan por un notable incremento en los espacios comunes, si bien, cuenta con una gran diversificación de funciones destinadas a dotar de servicios a la comunidad. El principal valor es la capacidad de crear actividades y usos múltiples, lo que obliga a una cultura de la gestión entre los comuneros. Queda claro que sin una participación de la comunidad en dicha gestión difícilmente puede funcionar este modelo, salvo que se externalice el mismo. Cabe destacar también que estas áreas comunes, en muchos casos, está abierto a la ciudad o el barrio, localizándose en planta baja, junto a los locales de actividad comercial.

\begin{tabular}{|c|c|c|c|c|c|c|}
\hline & $\begin{array}{l}\text { espacios } \\
\text { colectivos }\end{array}$ & vivienda & $\begin{array}{l}\text { construcción/ } \\
\text { mantenimiento }\end{array}$ & energia & movilidad $\uparrow \rightleftarrows$ & ใُ้ \\
\hline $\begin{array}{c}\text { Actividad } \\
\text { Residencial }\end{array}$ & \multirow{2}{*}{$\begin{array}{l}\text { - Articulación de espacios } \\
\text { privados, comunes y } \\
\text { públicos } \\
\text { - Lavanderia } \\
\text { - Sala fitness } \\
\text { - Espacios multifucionales } \\
\text { - Talleres de trabajo } \\
\text { - Cocina/catering } \\
\text { - Espacios recreativos } \\
\text { - Guarderia } \\
\text { - Espacio de trabajo } \\
\text { colaborativo } \\
\end{array}$} & \multirow{2}{*}{$\begin{array}{l}\text { - Formas } \\
\text { contemporáneas } \\
\text {-viviendas } \\
\text { convencionales } \\
\text {-viviendas en } \\
\text { clusters (4-21 } \\
\text { personas) }\end{array}$} & \multirow[t]{2}{*}{$\begin{array}{l}\text { - Bajo costo } \\
\text { - Modular } \\
\text { - Autoconstrucción de } \\
\text { las viviendas }\end{array}$} & \multirow[t]{2}{*}{$\begin{array}{l}\text {-Cogeneración } \\
\text { - Geotermia } \\
\text { - Paneles } \\
\text { fotovoltaicos }\end{array}$} & \multirow[t]{2}{*}{$\begin{array}{l}\text { limitación número } \\
\text { de ascensores }\end{array}$} & \multirow[t]{2}{*}{$\begin{array}{l}\text { - mixtificación } \\
\text { - diversidad social } \\
\text { - alquileres } \\
\text { escalonados }\end{array}$} \\
\hline $\mathbf{N}$ & & & & & & \\
\hline $\begin{array}{c}\text { Richmon } \\
d\end{array}$ & \multirow[t]{2}{*}{$\begin{array}{l}\text { Espacios comunitarios } \\
\text { dedicados a la } \\
\text { alimentación } \\
\text { Planta Baja: Restaurante } \\
\text { de propiedad y gestión } \\
\text { comunitaria + huerto de } \\
\text { producción ecológica }\end{array}$} & \multirow[t]{2}{*}{$\begin{array}{l}\text { Viviendas } \\
\text { trabajadodores + } \\
\text { realjojo de } \\
\text { residentes + } \\
\text { vivienda libre } \\
\text { alquiler+ } \\
\text { vivienda protegida } \\
\text { alquiler social }\end{array}$} & \multirow[t]{2}{*}{$\begin{array}{l}\text { Construcción de bajo } \\
\text { coste } \\
\text { Materiales y técnica } \\
\text { constructiva que } \\
\text { requieren escaso } \\
\text { mantenimiento }\end{array}$} & \multirow[t]{2}{*}{$\begin{array}{l}\text { Envolvente de alta } \\
\text { eficiencia energética } \\
\text { Aprovechamiento } \\
\text { recursos naturales } \\
\text { (soleamiento, aguas } \\
\text { pluviales, compostaje } \\
\text { de residuo orgánico) }\end{array}$} & \multirow[t]{2}{*}{$\begin{array}{l}\text {-amplias zonas } \\
\text { aparcamiento } \\
\text { bicicleta } \\
\text { - puesto de coche } \\
\text { compartido (sistema } \\
\text { municipal) }\end{array}$} & \multirow[t]{2}{*}{ diversidad social } \\
\hline $\mathbf{N}$ & & & & & & \\
\hline Covicivi & \multirow{3}{*}{$\begin{array}{l}\text { Espacios de uso público: } \\
\text { - escuela taller para el } \\
\text { barrio } \\
\text { - locales comerciales } \\
\text { - salón comunitario }\end{array}$} & \multirow{3}{*}{$\begin{array}{l}11 \text { viviendas } \\
\text { rehabilitadas } \\
8 \text { viviendas de } \\
\text { nueva planta } \\
\text { viviendas de } \\
\text { autoconstrucción }\end{array}$} & \multirow{3}{*}{$\begin{array}{l}\text { aportación de horas } \\
\text { de trabajo por los } \\
\text { cooperativistas(coope } \\
\text { rativa de ayuda } \\
\text { mutua) } \\
\text { banco de tiempo }\end{array}$} & & & \multirow{3}{*}{$\begin{array}{l}\text { aprendizaje de los } \\
\text { propietarios sobre } \\
\text { el valor patrimonial } \\
\text { de la edificación } \\
\text { formación e } \\
\text { inserción laboral } \\
\text { identidad cultural }\end{array}$} \\
\hline $\begin{array}{l}\text { Actividad } \\
\text { Escuela } \\
\text { Taller }\end{array}$ & & & & & & \\
\hline N/R & & & & & & \\
\hline
\end{tabular}

Figura 4. Cuadro comparativo de las diferentes cooperativas analizadas

\begin{tabular}{|c|c|c|c|c|c|c|}
\hline & $\begin{array}{l}\text { espacios } \\
\text { colectivos }\end{array}$ & vivienda & $\begin{array}{l}\text { construcción/ } \\
\text { mantenimiento }\end{array}$ & $x^{2}$ & movilidad $\uparrow \rightleftarrows$ & 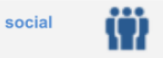 \\
\hline $\begin{array}{l}\text { Los } \\
\text { Milagros } \\
\text { (4) } \\
\text { Actividad } \\
\text { Residencial } \\
\mathbf{N}\end{array}$ & $\begin{array}{l}\text { Comedor } \\
\text { Lavanderia } \\
\text { Huerto ecológico } \\
\text { Espacios dedicados a } \\
\text { medicina y fisioterapia } \\
\text { Espacios terapia } \\
\text { ocupacional } \\
\text { Piscina y y espacios } \\
\text { recreativos }\end{array}$ & $\begin{array}{l}\text { socios residentes + } \\
\text { socios temporales }\end{array}$ & $\begin{array}{l}\text { personal de } \\
\text { mantenimiento } \\
\text { sufragado por la } \\
\text { cooperativa de } \\
\text { consumo }\end{array}$ & & $\begin{array}{l}\text { servicio de } \\
\text { transporte } \\
\text { (microbús) de } \\
\text { propiedad y gestión } \\
\text { cooperativas }\end{array}$ & $\begin{array}{l}\text { agrupación } \\
\text { ciudadanos sénior } \\
\text { unión de personas } \\
\text { con necesidades } \\
\text { semejantes (coop. } \\
\text { de consumo) } \\
\text { actividades } \\
\text { orientadas a sector } \\
\text { de población } \\
\text { especifico }\end{array}$ \\
\hline 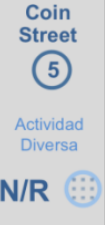 & $\begin{array}{l}\text { Espacios públicos } \\
\text { Parques y jardines } \\
\text { Espacios comunitarios } \\
\text { Centro civico } \\
\text { Centro deportivo } \\
\text { Espacios culturales }\end{array}$ & $\begin{array}{l}4 \text { cooperativas de } \\
\text { vivienda [lroko, } \\
\text { Mulberry, Palm, } \\
\text { Redwood] } \\
1 \text { promoción de } \\
\text { vivienda libre en } \\
\text { desarrollo (Doon } \\
\text { Street }\end{array}$ & $\begin{array}{l}\text { Gestionados por la } \\
\text { empresa Coin Street } \\
\text { Community Builders }\end{array}$ & & $\begin{array}{l}\text { Dotación de } \\
\text { aparcamientos que } \\
\text { financian parte de la } \\
\text { operación }\end{array}$ & $\begin{array}{l}\text { economía social } \\
\text { creada desde la } \\
\text { participación } \\
\text { beneficios de CSCB } \\
\text { destinados a la } \\
\text { mejora del barrio } \\
\text { amplia oferta de } \\
\text { actividad para la } \\
\text { propia comunidad y } \\
\text { sus sectores de } \\
\text { población }\end{array}$ \\
\hline $\begin{array}{l}\text { R-Urban } \\
\text { Actividad } \\
\text { Restauración } \\
\text { N } \because 3\end{array}$ & $\begin{array}{l}\text { Huertos urbanos intensivos } \\
\text { y laboratorio agricola } \\
\text { Talleres de reciclaje y de } \\
\text { formación. } \\
\text { Espacios para la } \\
\text { celebración de eventos } \\
\text { Laboratorio de tecnologia } \\
\text { asequible }\end{array}$ & $\begin{array}{l}\text { Edificio de } 7 \\
\text { viviendas en } \\
\text { régimen de } \\
\text { cooperativa con } \\
\text { fuerte carga } \\
\text { ecológica (fase de } \\
\text { proyecto). Proyecto } \\
\text { de rehabilitación del } \\
\text { barrio }\end{array}$ & $\begin{array}{l}\text { Especial atención al } \\
\text { reciclaje }\end{array}$ & $\begin{array}{l}\text { Energia renovable en } \\
\text { el edificio de } \\
\text { viviendas. Reciclaje } \\
\text { de agua. } \\
\text { Prácticas de } \\
\text { resiliencia urbana }\end{array}$ & $\begin{array}{l}\text { Entornos de acción } \\
\text { con radios que } \\
\text { permiten movilidad } \\
\text { peatonal o en } \\
\text { bicicleta }\end{array}$ & $\begin{array}{l}\text { Experiencia botton- } \\
\text { up. Fomento de la } \\
\text { resiliencia urbana } \\
\text { Estrategias de } \\
\text { consumo y } \\
\text { producción. } \\
\text { Jardín comunitario } \\
\text { entendido como } \\
\text { lugar de relación de } \\
\text { los residentes } \\
\end{array}$ \\
\hline
\end{tabular}




\title{
WPSReview International on Sustainable
}

\author{
Housing and Urban Renewal (RI-SHUR)
}

Las viviendas se caracterizan por la diversidad tipológica y de renta de sus ocupantes, lo que evita la gentrificación. También cabe destacar la mezcla de unidades residenciales (copropietarios) junto a una oferta de alojamiento no residencial (alquiler para personas, familias o grupos grandes). En algunos casos existen tipologías muy definidas, junto a otras más abiertas.

En la construcción y el mantenimiento de la obra nueva prevalece el criterio de diseño con materiales de mantenimiento fácil y económico. Por otra parte, se favorece el diseño modular y la autoconstrucción. En este último caso se obliga a una formación previa de los socios, o bien a la utilización de sistemas constructivos de montaje sencillo. La incorporación de los socios en el posterior mantenimiento de los edificios puede ser facilitada y gestionada a través del banco de tiempo, donde cada socio pone al servicio de la comunidad sus habilidades y puede recibir contraprestaciones sin necesidad de intercambio monetario.

Los modelos energéticos de los casos de estudio sólo se plantean en los edificios de obra nueva, o bien, en las cooperativas específicamente pensadas para su producción o gestión. La preocupación sobre la envolvente energética ocupa un importante papel, sobre todo en experiencias con climas más nórdicos, que se traslada en la utilización de sistemas de producción de calor solar, geotérmico o de cogeneración.

En la movilidad, curiosamente se apuesta por una reducción del número de plazas de aparcamiento, a favor de ceder espacio para el aparcamiento de bicicletas, o facilitar la utilización del car-sharing, reservando zonas especialmente para ello. También destaca la posibilidad de que los socios financien sus propios medios de transporte colectivo, sobre todo en aquellos sectores con características especiales (personas con diversidad funcional).

Sobre el impacto socio-económico, aunque desde diferentes enfoques, todos ellos plantean un importante beneficio social desde el acceso a la vivienda, la protección del patrimonio, la defensa de la población local o la protección del medio ambiente. De una forma directa o indirecta, todas las experiencias están dirigidas a la creación de una economía social paralela, que constituye un importante avance sobre el desarrollo de políticas meramente finalistas y centradas en un único objetivo. En este sentido, la política de vivienda se ha dirigido a la creación de un parque de vivienda, cuyo impacto en el sector de la construcción era importante durante su ejecución, pero que se resentía en períodos de crisis del sector.

Finalmente se puede observar que en las experiencias de co-vivienda subyacen dos estructuras superpuestas: una estructura básica compuesta por los socios de la cooperativa, constituyen la estructura más estable y dota de significado a la misma; frente a una estructura periférica, formada por no socios o socios temporales, que se benefician de los servicios generados por la actividad y que la dotan de un enriquecimiento social y económico, que evita la degeneración de la cooperativa en un gueto social.

Indudablemente la aportación del sistema de co-vivienda no acaba en la competitividad que pueda suponer para la comunidad. Las personas que viven en este

WPS RI-SHUR, nำ, 2016, vol.1, ISSN: 2387-1768 


\title{
WPSReview International on Sustainable
}

\author{
Housing and Urban Renewal (RI-SHUR)
}

tipo de cooperativas lo hacen sabiendo que se rigen por unos principios de solidaridad y democracia, por lo que este tipo de experiencias dan como resultado colectivos con una fuerte cohesión social, donde se crean de manera casi natural redes de apoyo mutuo, y se produce una vida social más armonizada de lo que suele ser habitual en la vivienda colectiva. Frente a una parte de la sociedad, ciertamente importante, que considera la vida en comunidad como un mal menor, y optan por el ideal de la casa suburbana "sin vecinos", que representa el ideal individualista de la ciudad del urban sprawl devoradora de territorio y recursos; existe, sin duda, otro importante colectivo que entiende la vida comunitaria como un foco de diversidad y el espacio urbano como un lugar de intercambio. Esta situación de partida posibilita la idea de aprovechar todo el potencial de la comunidad, entendiendo el hábitat colectivo como una ventaja más que como un inconveniente, por eso la co-vivienda posibilita que ese intercambio, a escala doméstica, se traslade al propio hábitat del individuo.

Esta apuesta por lo comunitario se suele establecer en los modelos cooperativos estudiados en tres niveles:

\section{El uso del espacio}

El modo de vida asociado a la vivienda colaborativa, como cualquier otro, se refleja en la estructura espacial y arquitectónica de los conjuntos de viviendas en los que se desarrolla. En el modelo tradicional de la comunidad de vecinos, se tiende a que el espacio de lo común se reduzca al mínimo, al ser considerado una especie de carga inevitable que debe ser sufragada por todos los vecinos. En un modelo de co-vivienda esta tendencia se invierte: más vida en común requiere lógicamente más espacios de relación y actividad, y por ello lo habitual es encontrar conjuntos residenciales con amplias zonas comunes e incluso con edificaciones completas destinadas al uso colectivo.

\section{El uso del tiempo}

Los habitantes de una agrupación de co-housing con frecuencia también extienden su colaboración a la comunidad donando parte de su tiempo para el beneficio de esta. Así, es frecuente que en el seno de estas asociaciones se realicen numerosas actividades colectivas de carácter voluntario, desde jardinería u horticultura ecológicas hasta talleres formativos diversos.

También puede complementarse o sustituirse la aportación económica de algunos de los cooperativistas mediante una retribución en especie, aportando horas de trabajo en aspectos de utilidad para la comunidad, desde tareas de mantenimiento hasta la gestión económica y administrativa de la cooperativa, por lo que el modelo puede asimilar aspectos propios de las cooperativas de trabajo asociado.

\section{El uso de los recursos económicos}

Como en cualquier comunidad de vecinos, en la co-vivienda se establece una cuota de participación para cada uno de los socios. La mayor diferencia en este aspecto entre WPS RI-SHUR, nํ, 2016, vol.1, ISSN: 2387-1768 


\title{
WPSReview International on Sustainable
}

\author{
Housing and Urban Renewal (RI-SHUR)
}

un modelo y otro radica en que en el co-vivienda, los elementos comunes tienen más relevancia, por lo que necesitarán mayores aportaciones económicas. A cambio, la cuota de pertenencia a la comunidad -es decir, la adquisición del derecho de uso de una vivienda- suele tener un coste relativamente bajo, además de disponer de un abanico de servicios comunes por lo general de cierta amplitud. A menudo las comunidades de co-vivienda establecen también cuotas de mayor o menor cuantía en función de la implicación que cada socio quiera tener en cada una de los espacios, actividades o servicios comunes.

Lógicamente, la implicación del cooperativista en estos tres niveles -salvo en lo relativo a cuotas de derecho de uso o de alquiler de vivienda- es absolutamente voluntaria. De otra manera, el hecho de habitar un espacio de co-vivienda podría llegar a suponer una carga para el cooperativista y provocar efectos contrarios a los que pretende conseguir.

\section{4.-Debate. La cooperativa vecinal como modelo de rehabilitación.}

En la traslación de este modelo a la recuperación de barriadas, pobladas en su mayor parte por personas con ingresos económicos muy ajustados, los modelos de cooperativa y de co-vivienda deben intentar dar un paso más. Dado que el principal problema al que se enfrenta el desafío de la rehabilitación de barriadas es la financiación de las intervenciones que deben ser acometidas, el interrogante que se abre es ¿puede el sistema cooperativo generar una economía social capaz de producir una actividad económica que permita financiar el proyecto? ¿pueden las fuerzas vivas del colectivo que habita una barriada utilizarse para beneficio del propio barrio, constituyendo un foco de revitalización urbana y una fuente de ingresos y de empleo para sus habitantes? Se trata de intentar estudiar los posibles mecanismos para que esa implicación social en tres niveles sirva para generar recursos para la propia barriada y sus vecinos. Dicho de otro modo, que el uso del espacio, del tiempo y de los recursos económicos de los ciudadanos sumen fuerzas para crear un panorama económico que haga viable la mejora del entorno urbano de la barriada y de los edificios que la componen. 


\section{WPSReview International on Sustainable}

\section{Housing and Urban Renewal (RI-SHUR)}

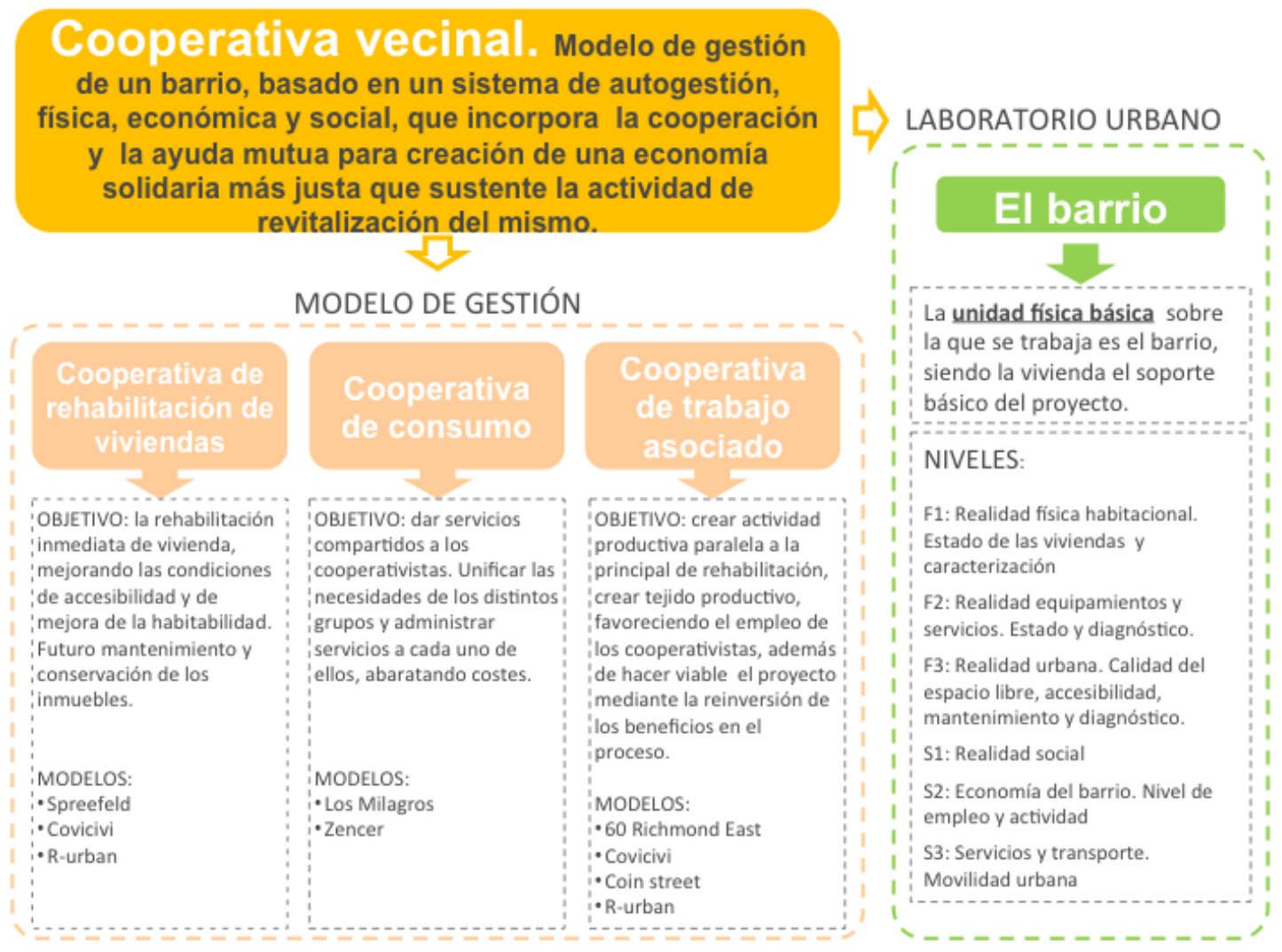

Figura 6. La Cooperativa Vecinal como modelo de gestión. Estrategia de intervención en una barriada prototipo.

Se propone el concepto de cooperativa vecinal como propuesta de un nuevo modelo de cooperativa, planteado como un instrumento que capacite a las barriadas y a otros entornos urbanos para gestionar y acometer las mejoras necesarias en el entorno físico y social repercutiendo en la mejora de la calidad de vida de sus ciudadanos. Para ello, se intentará incorporar al modelo en la medida de lo posible los aspectos más interesantes encontrados en cada uno de los modelos de éxito seleccionados, tomados como ejemplos que ilustran las características descritas que un modelo colaborativo como la co-vivienda puede aportar:

- Acceso a bienes y servicios en condiciones ventajosas

- Fomento de la economía social

- Solidaridad entre vecinos, puesta en valor de lo colectivo relacionado con lo habitacional

- Cohesión social, identidad y sentimiento de pertenencia al colectivo

- Diversidad social, enriquecimiento del individuo por la pertenencia a un grupo

- Diversidad funcional de los espacios; disposición de espacios comunitarios de escala superior a la doméstica por un coste relativamente bajo 


\title{
WPSReview International on Sustainable
}

\author{
Housing and Urban Renewal (RI-SHUR)
}

- Facilidad para saltar a la siguiente esfera, de lo colectivo a lo público. De la escala del edificio a la del vecindario o la del barrio

- Implantación de un modelo que puede ser objeto de la colaboración entre lo público y lo priva do en favor de la ciudad y los ciudadanos, en el que éstos adquieren un nivel participativo y emprendedor.

Los ejemplos de experiencias de éxito estudiados muestran posibles vías, caminos que una cooperativa vecinal puede abrir para conseguir estos objetivos. El caso de Coin Street ejemplifica la creación de una economía social duradera, estable y próspera partiendo de una base totalmente ciudadana (Velázquez, 2011). R-Urban muestra la eficiencia de las redes ciudadanas - que pueden ser apoyadas de manera eficiente por el uso de las tecnologías de la información-, para promover la autogestión de un área urbana intentando controlar los flujos de entrada y salida de recursos y bienes de consumo (VVAA, 2015c). La cooperativa uruguaya Covicivi nos acerca al modelo cooperativo de trabajo asociado o de ayuda mutua, donde los vecinos son capaces de convertirse en trabajadores cualificados al servicio de su propia empresa, a la vez que se implican en la puesta en valor y conservación del patrimonio urbano. En 60 Richmond East hemos podido observar una comunidad que comparte un modelo de negocio -en este caso un restaurante- utilizado como fuente de ingresos y como lugar de formación. La cooperativa andaluza Los Milagros nos muestra las ventajas de la comunidad como agrupación de consumidores y usuarios (VVAA, 2010), mientras que en Spreefeld encontramos un modelo de convivencia con modos de vida alternativos y con una gradación espacial desde el ámbito privado de cada cooperativista hasta la escala urbana. Por último, la cooperativa Zencer, como cooperativa de consumidores, puede posicionarse en mercados tan complejos como el de la energía y abrir nuevos nichos de negocio, aportando también un esfuerzo para intentar cambiar el modelo energético del que dependen nuestras ciudades.

\section{Conclusión}

Por un lado, cabe destacar la existencia en general de escasas experiencias de rehabilitación de viviendas a través del sistema cooperativo, así como la escasez de bibliografía al respecto, donde muchos casos de co-vivienda/co-housing están poco documentados, reduciéndose a breves referencias en soporte web (revistas online, blogs, ...).

Las ventajas de un sistema vecinal cooperativo son claras, el acceso a una vivienda digna, el ahorro, la protección del entorno, la solidaridad entre generaciones y vecinos, y con ello intentar evitar algunas situaciones de exclusión social, soledad y abandono. En realidad, es favorecer una plataforma que sea capaz de recuperar los valores de la solidaridad vecinal, el respeto por el medio ambiente y la construcción de entornos saludables y accesibles.

La idea de gestión que introduce el modelo de cooperativa, se entiende desde una doble vertiente, como un mecanismo de rehabilitación en primera instancia, pero a su vez como un modo de gestión integral del mantenimiento y uso. Los espacios

WPS RI-SHUR, nำ, 2016, vol.1, ISSN: 2387-1768 


\section{WPSReview International on Sustainable \\ Housing and Urban Renewal (RI-SHUR)}

resultantes de este proceso no se acogen a los conceptos de propiedad o alquiler convencionales, ya que se suele optar por el modelo de cesión de uso.

Entendemos que las ventajas que introduce este nuevo método de gestión de comunidades, favorece la conservación y mantenimiento de las comunidades residenciales. Paralelamente, se puede crear un entorno apropiado para la creación de nuevas empresas, así como la reconversión de empresas de la construcción en empresas de conservación y mantenimiento integral de edificios.

\section{Referencias}

CAMERON, Jane et al (ed.), Profiles of a Movement: Co-operative Housing Around the World, ed. CECODHAS Housing Europe and ICA Housing, 2012

MORENO GONZÁLEZ, Aurora, Buenas prácticas, modelo y ambiente [en línea] $<$ http://www.fundacionpilares.org/modeloyambiente/docs/bbpp/BP7CooperativaLosMila gros.pdf > [consulta: 12 marzo 2015].

SCOTTHANSON, Chris, The cohousing handbook, ed. New Society, 2005

SUSCHEK-BERGER, Jürgen, COOPERATIVE REFURBISHMENT. Inclusion of Occupants and other Stakeholders in Sustainable Refurbishment Processes in MultiFloor Residential Buildings. Open house international Vol 35, No.2, Junio 2010

VALLÉS, R., ÁRIAS, D. Y SOLANAS, M. (2011). Habitar el patrimonio. La cooperativa de vivienda por ayuda mutua como medio de gestión y desarrollo edilicio y urbano, América patrimonio, (1).

VELÁZQUEZ VALORIA, Isabela y VERDAGUER VIANA-CÁRDENAS, Carlos. Regeneración urbana integral. Tres experiencias europeas innovadoras: Île de Nantes, Coin Street y Barrio de la Mina. Ed. SEPES Entidad Estatal de Suelo. Madrid, 2011

VVAA, So. Coop. And. Los Milagros: alternativa al cooperativismo de consumo, en Revista Fedeccon hoy, ํㅜ 5. Ed. Federación Andaluza de Empresas Cooperativas de Consumidores y Usuarios, abril-junio 2010

VVAA, Análisis urbanístico de barrios vulnerables en España. Sobre la vulnerabilidad urbana. Ministerio de Fomento, Instituto Juan de Herrera y DUYOT. [en línea] http://www.fomento.gob.es/NR/rdonlyres/C88DB66D-8669-497C-BEE4-

442AE027E2FB/111287/SOBRE_vulnerabilidad.pdf [consulta: 3 mayo 2015a]

VVAA, Citizen urbanism: economical scapes [en línea], $<$ http://viveroiniciativasciudadanas.net/2014/11/18/citizen-urbanism-economicalscapes/> [consulta: 16 marzo 2015b]

VVAA, R-urban Stratégie [en línea] < http://r-urban.net/accueil/ > [consulta: 22 abril 2015c].

VVAA, Las cooperativas de vivienda por ayuda mutua uruguayas. Una historia con quince mil protagonistas. IMM y Junta de Andalucía, 2008.

WPS RI-SHUR, n3, 2016, vol.1, ISSN: 2387-1768 